\title{
Adeno-tonsillectomy and rapid maxillary distraction in pre-pubertal children, a pilot study
}

\author{
Christian Guilleminault • Pierre-Jean Monteyrol • \\ Nelly T. Huynh • Paola Pirelli • Stacey Quo • Kasey Li
}

Received: 22 March 2010 /Revised: 12 August 2010/Accepted: 7 September 2010

(C) Springer-Verlag 2010

\begin{abstract}
Introduction When both narrow maxilla and moderately enlarged tonsils are present in children with obstructive sleep apnea, the decision of which treatment to do first is unclear. A preliminary randomized study was done to perform a power analysis and determine the number of subjects necessary to have an appropriate response. Thirtyone children, 14 boys, diagnosed with OSA based on clinical symptoms and polysomnography (PSG) findings had presence of both narrow maxillary complex and enlarged tonsils. They were scheduled to have both adeno-tonsillectomy and RME for which the order of treatment was randomized: group 1 received surgery followed by orthodontics, while group 2 received orthodontics followed by surgery. Each child was seen by an ENT, an orthodontist, and a sleep medicine specialist. The validated pediatric sleep questionnaire
\end{abstract}

C. Guilleminault $(\square) \cdot \mathrm{K}$. Li

Stanford University Sleep Medicine Division,

450 Broadway, Pavillon B,

Redwood City 94063 CA, USA

e-mail: cguil@stanford.edu

P.-J. Monteyrol

Polyclinique oto-laryngologie,

Bordeaux, France

N. T. Huynh

Dental Faculty University of Montreal,

Montreal, Canada

P. Pirelli

Tor-Vorgata University Orthodontic-Polyclinic,

Rome, Italy

S. Quo

Orthodontic Clinic,

Palo Alto, CA, USA and PSG were done at entry and after each treatment phase at time of PSG. Statistical analyses were ANOVA repeated measures and $t$ tests.

Results The mean age of the children at entry was $6.5 \pm$ 0.2 years (mean $\pm \mathrm{SEM}$ ). Overall, even if children presented improvement of both clinical symptoms and PSG findings, none of the children presented normal results after treatment 1 , at the exception of one case. There was no significant difference in the amount of improvement noted independently of the first treatment approach. Thirty children underwent treatment 2 , with an overall significant improvement shown for PSG findings compared to baseline and compared to treatment 1, without any group differences.

Conclusion This preliminary study emphasizes the need to have more than subjective clinical scales for determination of sequence of treatments.

Keywords Rapid maxillary expansion .

Adeno-tonsillectomy - Obstructive sleep apnea · Treatment . Power analysis

\section{Introduction}

At the 2006 World Congress on Sleep Apnea, a symposium on orthodontic treatment of pre-pubertal children with obstructive sleep apnea (OSA) chaired by Dr. Kasey Li demonstrated that rapid maxillary expansion(RME) had a role in the treatment of pediatric OSA [1-3]. Many questions were raised, one of which concerned the timing of such treatment when tonsils were also enlarged. When both narrow maxilla and moderately enlarged tonsils are present, the decision of which treatment to do first is unclear and treatment order is 
usually based on factors unrelated to the clinical presentation: often the first treatment applied was the one recommended by the specialist consulted first, commonly adeno-tonsillectomy if seen by an ear-nose and throat specialist (ENT) and RME if seen by an orthodontist. In other cases seen first in sleep clinics, the speed at which an appointment could be secured with one of the considered specialist was the deciding factor. Presence of enlarged tonsils are a long-term problem when orthodontic treatment is performed as mouth breathing still occurs, impacting the long-term gain obtained on nasal resistance with RME. A preliminary randomized study was planned and done to determine the number of subjects necessary to appropriately respond to the question. All subjects involved in the study are children with presence of both narrow maxillary complex and enlarged tonsils. Approval of protocol and of consent form was obtained from the ethic committee of the University Tor Vergata (Rome, Italy).

\section{Protocol}

Children had to be diagnosed with OSA based on clinical symptoms and polysomnography (PSG). They also must have both enlargement of tonsils (with or without adenoidal enlargement) and narrow maxilla associated with a high and narrow hard palate, as determined by both orthodontist and ENT. And the conclusion of the complete clinical evaluation was that the children needed to have both adeno-tonsillectomy and RME. At time of scheduling the two treatments, the order of treatment was to be randomized: group 1 receiving surgery followed by orthodontics, while group 2 receiving orthodontics followed by surgery.

\section{Randomization}

Randomization was based on a pre-established randomization table, but children consecutively seen were treated by pairs, i.e., if randomization table indicated that child \#1 was to be first treated with treatment A, child \#2 was automatically attributed to treatment B. Pair treatment decision allowed children treated in opposite order to have treatment performed for both of them in a relatively closed time period. It was also pre-established that if a child (or parents) decided to opt-out of the study after randomization but before beginning any treatment, the next identified child would be a replacement for the drop-out child with same treatment schedule. If a child dropped out between treatment 1 and 2, there was no replacement.

\section{Clinical evaluation}

Each child was seen by an ENT and an orthodontist and had a sleep/wake evaluation by a sleep medicine specialist. A validated pediatric sleep questionnaire [4] available in several languages was given at entry and after each treatment phase at time of PSG.

Evaluation Oral examination was performed to score tonsil and tongue position with respect to the position of the uvula using the Friedman et al. tonsil scale [5], the Friedman et al. tongue scale [6] and the Mallampati et al. scale [7]; a 3point scale was used to score of the inferior nasal turbinates from 1 normal to 3 (very enlarged) and a 0/1 scale was used to recognized presence/absence of a nasal septum deviation. A clinical dental evaluation was performed and orthodontists evaluated facial balance, tooth eruption, jaw morphology, and occlusion. The definition of narrow maxilla was made clinically by the experienced orthodontists. The decision to select maxillary expansion $(n=$ $27)$ or bimaxillary expansion $(n=4)$ was made by the treating orthodontists. RME was recommended using an appliance designed to be fixed to the teeth. Expansion rates were typically $0.25 \mathrm{~mm}$ per day, as measured at the appliance. Lower jaw expansion utilized either fixed or removal appliances with a slower rate of expansion. The decision to select rapid maxillary expansion or bimaxillary expansion was made by the treating orthodontist. Followup recordings were scheduled to be 4 weeks after ENT surgery, and 3 months after the end of the expansion with the device still in place.

\section{Polysomnography}

All children had a night of nocturnal recording at entry and after each treatment phase with monitoring of EEG, electroocculogram, chin and leg EMG, and one ECG lead. Respiration was always monitored with nasal cannula, mouth thermistor or thermo-couple, thoracic and abdominal bands, finger pulse-oxymetry, and neck microphone.

\section{Analysis}

Clinical and PSG data were collected for each child at time of each visit. All data were de-identified. PSG were scored following international criteria for determination of an apnea-hypopnea index (AHI) $[8,9]$ and following preestablished criteria to determine presence of respiratory events not included in the AHI to determine a respiratory disturbance index (RDI) [10]. 


\section{Statistical analysis}

A two-sample $t$ test analysis was done to compare both groups at entry to the study. An ANOVA repeated measures of PSG data was done between entry and treatment 1 between groups. As both groups were comparable for all measured variables, an ANOVA repeated measures was done between treatments to evaluate the effect of order of treatment. Finally, another ANOVA repeated measures was done between entry and end of study to evaluate the overall effect of both treatments. Following these ANOVA analyses, $t$ tests were done as post-hoc analyses when necessary.

\section{Results}

One subject dropped out after randomization but before beginning any treatment as parents had conflict between school vacation schedule and planed treatment. Thirty-one children, 14 boys, were involved in the study. Thirty children were seen at the same sleep clinic and by the same sleep medicine specialist and ENT working in collaboration with three orthodontists in the same practice. The last child was seen by another sleep medicine specialist-ENT-orthodontist team. The mean age of the children at entry was $6.5 \pm 0.2$ years (mean \pm SEM). All children were brought for medical evaluation for reported snoring (noted by parents) and clinical symptoms associated with OSA, more particularly daytime fatigue, poor school performances, inattention, and sleepwalking were variably distributed. There were 16 children in group 1 and 15 children in group 2. One child in group 2 only completed treatment \#1, as PSG results were normalized and clinical symptoms had disappeared, thus for treatment \#2, group 1 had 16 children and group 2 had 14 children. Eighteen children had tonsils scored $2+$ and thirteen as $3+$. Twenty-eight children were scored as 3 and three as 4 at the Friedman et al. tongue maneuver [6] with similar scores at the Mallampati maneuver [7]. All children had a higher and narrower than expected hard palates and presence of dental crowding. Entry PSG results showed that all 31 children had OSA with an overall AHI of $11.8 \pm 0.6$, a RDI of $20.4 \pm 0.7$, and lowest $\mathrm{SaO} 2$ of $92.3 \pm 0.3$. Thus, after evaluation by the teams of specialists collaborating together for a long

Table 1 Studied groups and results after each treatment

\begin{tabular}{|c|c|c|c|c|c|c|c|c|c|}
\hline \multirow[t]{2}{*}{ Variables } & \multicolumn{2}{|l|}{ Baseline } & \multirow[t]{2}{*}{$p$ value } & \multicolumn{2}{|c|}{ Treatment 1} & \multirow[t]{2}{*}{$p$ value } & \multicolumn{2}{|l|}{ Treatment 2} & \multirow[t]{2}{*}{$p$ value } \\
\hline & Group 1 & Group 2 & & $\begin{array}{l}\text { Group } 1 \\
\text { surgery }\end{array}$ & $\begin{array}{l}\text { Group } 2 \\
\text { orthodontics }\end{array}$ & & $\begin{array}{l}\text { Group } 1 \\
\text { orthodontics }\end{array}$ & $\begin{array}{l}\text { Group } 2 \\
\text { surgery }\end{array}$ & \\
\hline \multicolumn{10}{|l|}{ Sleep variables } \\
\hline $\mathrm{TST}, \min$ & $431.3 \pm 4.1$ & $423.1 \pm 2.2$ & 0.09 & $429.1 \pm 5.6$ & $425.1 \pm 5.3$ & $\begin{array}{l}0.51 \mathrm{a} \\
0.99 \mathrm{~b} \\
0.28 \mathrm{c}\end{array}$ & $445.7 \pm 8.7$ & $438.7 \pm 4.7$ & $\begin{array}{l}0.72 \mathrm{a} \\
0.00 \mathrm{~b} \\
0.51 \mathrm{c}\end{array}$ \\
\hline REM, \% & $18.6 \pm 0.4$ & $18.9 \pm 0.3$ & 0.60 & $20.5 \pm 0.3$ & $20.1 \pm 0.2$ & $\begin{array}{l}0.13 \mathrm{a} \\
0.00 \mathrm{~b} \\
0.81 \mathrm{c}\end{array}$ & $22.0 \pm 0.2$ & $21.9 \pm 0.3$ & $\begin{array}{l}0.32 \mathrm{a} \\
0.00 \mathrm{~b} \\
0.45 \mathrm{c}\end{array}$ \\
\hline \multicolumn{10}{|c|}{ Sleep apnea variables } \\
\hline AHI, events/h & $12.5 \pm 0.8$ & $11.1 \pm 0.7$ & 0.20 & $4.9 \pm 0.6$ & $5.4 \pm 0.6$ & $\begin{array}{l}0.15 \mathrm{a} \\
0.00 \mathrm{~b} \\
0.53 \mathrm{c}\end{array}$ & $0.9 \pm 0.3$ & $0.9 \pm 0.3$ & $\begin{array}{l}0.16 \mathrm{a} \\
0.00 \mathrm{~b} \\
0.49 \mathrm{c}\end{array}$ \\
\hline RDI, events/h & $21.3 \pm 1.0$ & $19.5 \pm 1.0$ & 0.22 & $8.0 \pm 0.7$ & $7.9 \pm 0.5$ & $\begin{array}{l}0.27 \mathrm{a} \\
0.00 \mathrm{~b} \\
0.29 \mathrm{c}\end{array}$ & $1.6 \pm 0.6$ & $1.7 \pm 0.8$ & $\begin{array}{l}0.92 \mathrm{a} \\
0.00 \mathrm{~b} \\
0.95 \mathrm{c}\end{array}$ \\
\hline Lowest $\mathrm{SaO} 2 \%$ & $92.1 \pm 0.5$ & $92.5 \pm 0.4$ & 0.53 & $95.2 \pm 0.3$ & $95.9 \pm 0.3$ & $\begin{array}{l}0.65 \mathrm{a} \\
0.00 \mathrm{~b}\end{array}$ & $98.0 \pm 0.2$ & $97.6 \pm 0.3$ & $\begin{array}{l}0.004 \mathrm{a} \\
0.00 \mathrm{~b}\end{array}$ \\
\hline
\end{tabular}

Results obtained from 31 pre-pubertal children with OSA with either rapid maxillary expansion or adeno-tonsillectomy as treatment 1, with reverse treatment as second step. Independent samples $t$ tests were done to compare entry polysomnography results between groups. ANOVA repeated measures were done to compare baseline to treatment 1 results between groups, and to compare treatments 1 to 2 results between groups TST total sleep time, $A H I$ apnea-hypopnea index, $R D I$ respiratory disturbance index, $\mathrm{SaO} 2$ oxygen saturation, $a$ effect of treatments per group, $b$ effect of treatments, $c$ effect of group 
time, all children were felt to have maxillary $(n=27)$ or maxillary and mandibular $(n=4)$ involvement and that adeno-tonsillectomy and orthodontic maxilla-mandibular expansion would be needed considering the clinical presentation and the OSA symptoms and findings. The results of the entry PSGs of each treatment group are presented in Table 1. As can be seen there was no significant difference between sleep and respiratory variables between groups at entry.

Overall, even if children presented improvement of both clinical symptoms and PSG findings, none of the children presented normal results after treatment one (Table 1) at the exception of one case. There was no significant difference in the amount of improvement noted independently of the first treatment approach. Thirty children underwent treatment 2, with an overall significant improvement shown for PSG findings compared to baseline and compared to treatment 1 (Table 1). Although lowest $\mathrm{SaO} 2$ showed a significant interaction between treatments and groups $(p=$ 0.004 ), this difference was not seen with further analyses looking at each groups separately. Both groups showed improvement between treatments 1 and $2(p \leq 0.006$, paired $t$ tests), but without significant differences between groups ( $p \geq 0.1$, two-sample $t$ tests).

When individual results were examined, there was resolution of all complaints and normal PSG in one child following treatment 1 alone, after both treatments there was resolution of all problems in 28 children, and improvement but persistence of abnormal PSG findings in two individuals who presented respectively: at entry an AHI of 8, RDI of 25.2 , lowest $\mathrm{SaO} 2$ of $93 \%$ and an AHI of 16, RDI of 26 and lowest $\mathrm{SaO} 2$ of $89 \%$, and after both treatments an AHI of 5, RDI of 11.8, lower $\mathrm{SaO} 2$ of $95 \%$ and an AHI 5.1, RDI of 10 , and lower $\mathrm{SaO} 2$ of $96 \%$, indicating the need for further treatment. The measurement of the maxillary intermollar distance for the 31 studied children showed a mean increase of $3.68 \pm 0.53 \mathrm{~mm} 3$ months after termination of expansion.

This study was constructed to perform a power analysis to determine number of patients needed for a valid response and thus was not powered to give a response to the question: using $\mathrm{G}$ power analysis (with $\alpha$ error $=0.05$ ) and power 0.95 the minimum number of subjects would be 111, but if we use the Kirk sample size strict criteria, the total sample size is 134 if we assume a prevalence of pediatric OSA of $1 \%$ and 395 subjects with a $3 \%$ prevalence.

\section{Discussion}

In our small group of children, there was no significant difference between the group beginning with orthodontic treatment and the one beginning with surgical treatment after the first phase of treatment. Most children needed both treatments to have complete resolution of their symptoms and normalization of PSG. The orthodontic evaluation was done again 3 months after end of the RME with the device still in place and at that time there was retention of the gain obtained at end of distraction. This was a preliminary study performed to determine the power needed to have a valid response to the question. Based on the results obtained, using the kirk sample size strict criteria, a larger number of subjects (minimum 137) should be involved in the study. Two facts come out from these calculation based on this study: in many cases it will not matter which treatment is started first as both will be needed, but in some cases there will be the possibility that one treatment done first resolves the problem. Our preliminary study did not indicate which criteria would be useful to identify these children. Decision on size of tonsils is based on the Friedman et al. scale[6]. It is a visual subjective scale and it appreciates tonsils in a horizontal plane, but ignores the vertical extension. Furthermore, if abnormal narrowness of the maxilla is easy to suspect, the exact degree of narrowness present is often a difficult determination. Frequently, there is an interaction between skeletal structures and enlargement of soft tissues in the upper airway when abnormal breathing during sleep is detected. The different tests performed can suggest dominance of a problem. Often, determination of the roles of each component will be difficult in this group of patients, as seen here, suggesting the need to perform both treatments, and requesting at least the performance of a PSG after initial treatment before declaring a child "cured". This preliminary study emphasizes also the need to have more than subjective clinical scales for determination of which treatment should be performed first, perhaps addition of a frontal cephalogram in the selection criteria may be helpful.

Acknowledgment We thank the statistical expertise received from the University of Montreal Dental School (Montreal, Canada), and from Dr. YS Huang.

\section{References}

1. Pirelli P, Saponara M, Guilleminault C (2004) Rapid maxillary expansion in children with obstructive sleep apnea syndrome. Sleep 27:761-766

2. Villa MP, Malagnola C, Pagani J, Montesano M, Rizzoli A, Guilleminault C, Ronchetti R (2007) Rapid maxillary expansion in children with obstructive sleep apnea syndrome: 12 months follow-up. Sleep Med 8:128-134

3. Cistulli PA, Palmisano RG, Pools MD (1998) Treatment of obstructive sleep apnea syndrome by rapid-maxillary-expansion. Sleep 21:11-15

4. Chervin RD, Hedger K, Dillon JE, Pituch KJ (2000) Pediatric sleep questionnaire (PSQ): validity and reliability of scales for 
sleep disordered breathing, snoring, sleepiness, and behavioural problems. Sleep Med 1:21-32

5. Friedman M, Ibrahim H, Bass L (2002) Clinical staging for sleepdisordered-breathing. Otolaryngol Head Neck Surg 127:13-21

6. Friedman M, Tanyeri H, La Rosa M, Landsberg R, Vaidyanathan K, Pieri S, Caldarelli D (1999) Clinical predictors of obstructive sleep apnea. Laryngoscope 109:1901-1907

7. Mallampati SR, Gatt S, Gugino SL, Desai S, Waraksa B, Freiberger D, Liu P (1985) A clinical sign to predict difficult tracheal intubation: a prospective study. Can Anaesth Soc J $32: 429-434$
8. Rechtschaffen A, Kales A (eds) (1968) A manual of standardized terminology, techniques and scoring system for sleep stages of human subjects. Brain Research Institute, Los Angeles

9. The Sleep Disorders Atlas Task Force of the American Sleep Disorders Association (1992) EEG arousals: scoring rules and examples: a preliminary report of the Sleep Disorders Atlas Task Force of the American Sleep Disorders Association. Sleep 15:173-184

10. Guilleminault C, Li K, Khramtsov A, Pelayo R, Martinez S (2004) Breathing patterns in prepubertal children with sleep-relatedbreathing disorders. Arch Pediatr Adolesc Med 158:153-161 